\title{
Chemomechanical mapping of biopolymers and cells
}

\author{
K.J. Van Vliet, C.A. Tweedie, M.T. Thompson and S.Y. Lee \\ Department of Materials Science and Engineering, Massachusetts Institute of Technology \\ Cambridge, MA 02139 USA
}

Nanoscale mechanical characterization of surfaces and structures is rapidly advancing, particularly in the analysis of compliant, viscoelastic materials such as engineered polymers, tissues, cells, and molecules. Here, we discuss several recent applications of our mechanical analysis in these systems, including mapping of combinatorial polymer libraries created for biocompatibility or biodegradability; fundamental understanding of the structure-property connections in engineered and biomimetic polymer thin films; and ligand-binding avidity of molecular receptors on biological cell surfaces. In each of these examples, pointwise acquisition of pico- to nanoscale force-displacement responses enables visualization of the material surface, where image contrast is based on either mechanical and/or chemical interactions. This capability is particularly useful in mapping the environmentally induced changes in structural / mechanical features of the surface, as we will discuss in the context of living biological cells.

The mechanical behavior of polymers is typically analyzed with recourse to phenomenological spring-and-dashpot models that predict experimentally observed deformation, but do not relate uniquely to the polymer microstructure that could impact the rationale design of polymers for applications ranging from dielectric coatings to biological cell substrata. One approach to determine structure-property correlations is the rapid analysis of discrete combinatorial libraries, spots of nL-scale volume and $\mu \mathrm{m}$-scale thickness for which composition is varied systematically. Here, we demonstrate high throughput mapping of mechanical stiffness in such libraries via

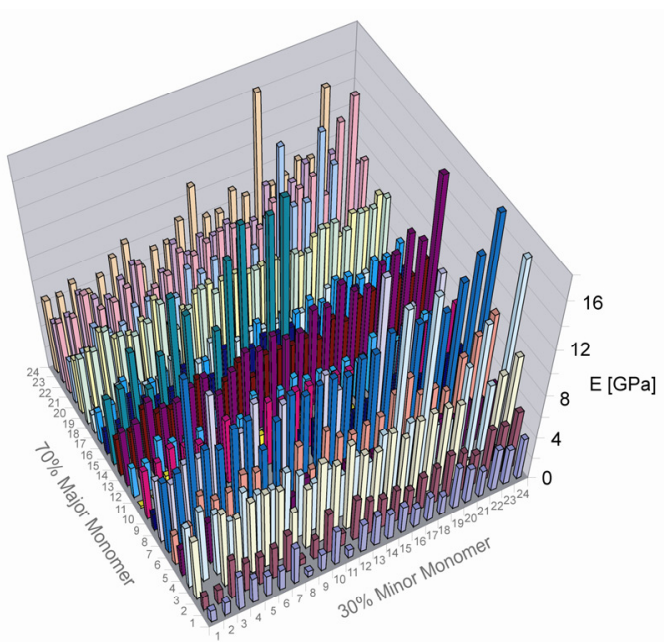
instrumented nanoindentation. Figure 1 shows the effective elastic modulus for 576 member UV-crosslinked polyacrylate library that was synthesized within $24 \mathrm{hrs}$ and then mechanically analyzed within $24 \mathrm{hrs}$ [1]. We have extended this approach to optimize the mechanical properties of biodegradable polymers.

The mechanical characterization of hydrated polymer films is of increasing interest because growing experimental evidence suggests that cell attachment and growth 
Figure 2. (A) Nominal elastic modulus $E$ as a function of polymer electrolyte multilayer (PEM) assembly $\mathrm{pH}$; polymers characterized via SPM at neutral $\mathrm{pH}$. (B) Total number of viable cells attached to PEMs as a function of assembly $\mathrm{pH}$ and correlative with PEM stiffness. TCPS = tissue culture-treated polystyrene, as described in [3].
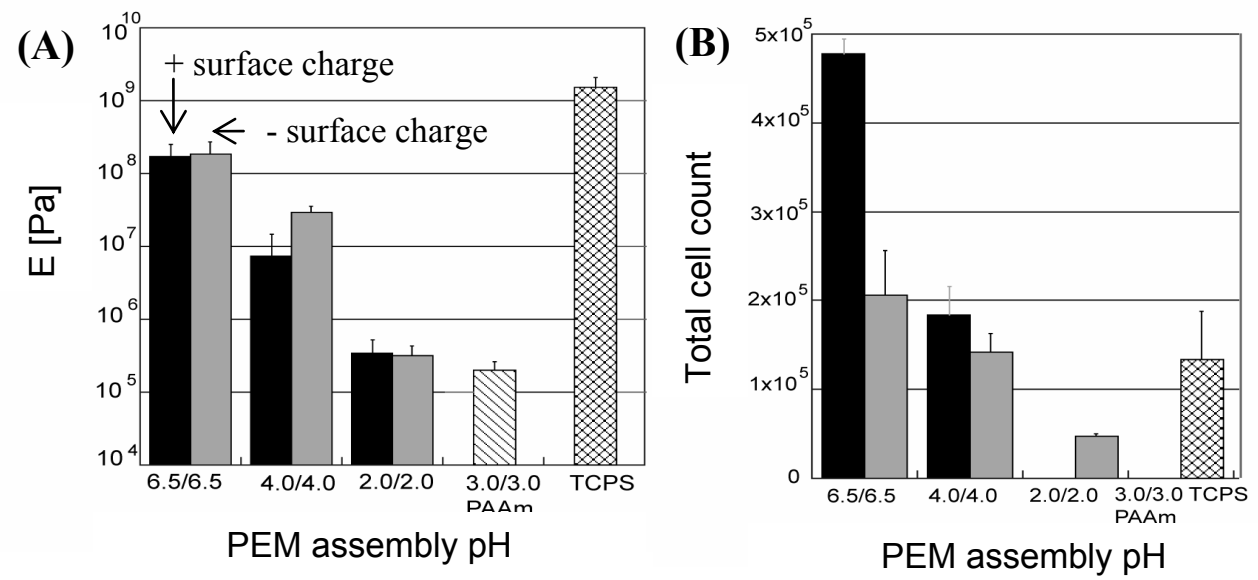

on such materials correlates directly with mechanical compliance [2, 3]. We apply scanning probe microscopy-enabled indentation to demonstrate that the compliance of hydrated polyelectrolyte multilayer films of $100 \mathrm{~nm}$ thickness can be varied by orders of magnitude to directly control the attachment of both eukaryotic and prokaryotic cells to these polymers (e.g., Fig. 2). Finally, we show that scanning probe microscopy can also be applied to the mechanical mapping of cell surface structure and molecular landscapes. The force required to separate a cantilevered probe-bound ligand and a cell surface receptor can be utilized to produce image contrast to map receptor distribution and binding kinetics (Fig. 3). Together, these probe microscopy/spectroscopy analyses of (bio)polymeric mechanics allow us to image and to understand modulation of cell structure and function via mechanical cues.
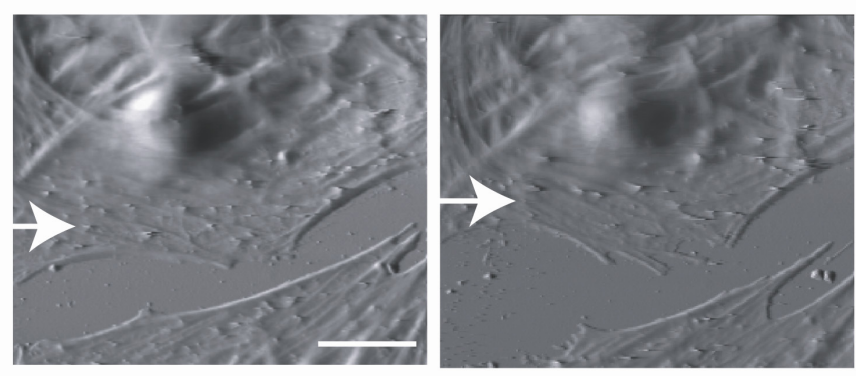

Figure 3. Functionalized force imaging of living endothelial cells. Scanning probe microscopy deflection image of cells shows discrete locations of probe-binding (e.g., arrow) when probe is functionalized with ligand (left), which decrease in number during competitive inhibition (right, $\mathrm{t}=30$ $\min )$. Scalebar $=10 \mu \mathrm{m}$.

1. Tweedie CA, Anderson DG, Langer R and Van Vliet KJ. Combinatorial material mechanics: High-throughput polymer synthesis and nanomechanical screening. Advanced Materials 17: 2599-2604 (2005).

2. Pelham RJ Jr. and Wang Y.-L. Cell locomotion and focal adhesions are regulated by substrate flexibility. Proc. Natl. Acad. Sci. USA 94:13661-13665 (1997).

3. Thompson MT, Berg MC, Tobias IS, Rubner MF and Van Vliet KJ. Tuning compliance of polyelectrolyte multilayers to modulate cell adhesion. Biomaterials 26: 6836-6845 (2005). 